\title{
Paradoxical proliferative potential of iron (II) sulphate on cancer cells after the 3-(4,5-dimethylthiazol-2-yl)-5- (3-carboxymethoxyphenyl)-2-(4-sulfophenyl)-2H- tetrazolium (MTS) assay
}

\author{
STANTON HON LUNG KOK ${ }^{1}$, ROBERTO GAMBARI ${ }^{2}$, CHUNG HIN CHUI ${ }^{1,3}$, FUNG YI LAU ${ }^{3}$, \\ GREGORY YIN MING CHENG ${ }^{3}$, PAUL BO SAN LAI ${ }^{4}$, WING SZE LAM ${ }^{1}$, ALBERT SUN CHI CHAN ${ }^{1}$, \\ CHOR HING CHENG ${ }^{1}$, IVY TUANG NGO TEO ${ }^{1}$, MICHAEL WING YIU YU ${ }^{1}$, \\ JOHNNY CHEUK ON TANG ${ }^{1}$, FILLY CHEUNG ${ }^{1}$ and RAYMOND SIU MING WONG ${ }^{3}$
}

\author{
${ }^{1}$ Lo Ka Chung Centre for Natural Anti-Cancer Drug Development (Laboratory B), Central Laboratory of the \\ Institute of Molecular Technology for Drug Discovery and Synthesis, State Key Laboratory of Chinese Medicine \\ and Molecular Pharmacology, Department of Applied Biology and Chemical Technology, The Hong Kong \\ Polytechnic University, P.R. China; ${ }^{2}$ ER-GenTech, Department of Biochemistry and Molecular Biology, \\ The University of Ferrara, Ferrara, Italy; Departments of ${ }^{3}$ Medicine and Therapeutics, and ${ }^{4}$ Surgery, \\ Prince of Wales Hospital, The Chinese University of Hong Kong, P.R. China
}

Received February 14, 2007; Accepted March 26, 2007

\begin{abstract}
There are several scientific approaches for the determination of cellular growth influences of known or novel substances under in vitro conditions, among which colourimetric absorption measurement is considered to be one of the convenient methods. [3-(4,5-dimethylthiazol-2yl)-5-(3-carboxymethoxyphenyl)-2-(4-sulfophenyl)-2Htetrazolium] (MTS) assay is one of the commonly used colourimetric absorption assays based on the ability of dehydrogenase from viable cells to produce the brown soluble formazan detectable at $490 \mathrm{~nm}$. Here we have tested the possible growth influence of iron (II) sulphate on two human cancer cell lines, the K562 chronic myelogenous leukaemia and T47D breast carcinoma cells, based on the MTS assay. We found that iron (II) sulphate possessed an inhibitory effect when added at 16 - to $125-\mu \mathrm{M}$ concentrations, but iron (II) sulphate became growth stimulatory when its concentration
\end{abstract}

Correspondence to: Dr Filly Cheung, Department of Applied Biology and Chemical Technology, The Hong Kong Polytechnic University, Yuk Choi Road, Hung Hom, Kowloon, Hong Kong, P.R. China

E-mail:bcfilly@inet.polyu.edu.hk

Dr Raymond S.M. Wong, Department of Medicine and Therapeutics, Prince of Wales Hospital, The Chinese University of Hong Kong, Ngan Shing Street, Sha Tin, N.T., Hong Kong, P.R. China

E-mail: rsmwong@cuhk.edu.hk

Key words: iron (II) sulphate, oxidation, proliferation was further increased to $1000 \mu \mathrm{M}$. In addition, a dosedependent increase in absorbance at the same wavelength was observed when we repeated the experiments without the addition of MTS and phenazine methosulfate. When we further repeated the cell growth determinations using adenosine triphosphate content assay for K562 and 3-(4,5-dimethylthiazol-2-yl)-2,5-diphenyltetrazolium bromide (MTT) assay for T47D, iron (II) sulphate showed a consistent dosedependent growth inhibitory effect. Morphological investigation after methylene blue staining clearly demonstrated that iron (II) sulphate, at a concentration of $1000 \mu \mathrm{M}$, is cytotoxic to T47D cells. Interestingly, a consistent increment for the absorbance at $490 \mathrm{~nm}$ was further observed with increased iron (II) sulphate concentration either in the presence or absence of MTS even in a cell-free environment. Thus we conclude that iron (II) sulphate is actually growth inhibitory and even cytotoxic at high concentrations towards the K562 and T47D cancer cells and the paradoxical proliferative activity of iron (II) sulphate on these two cancer cell lines using the MTS assay was solely due to the oxidation of initial pale green iron (II) to brownish iron (III) during incubation in the aqueous condition.

\section{Introduction}

High throughput current drug discovery depends on massive screening of chemical libraries against both extra- and intracellular molecular targets, to identify compounds with desired biological activities (1). In respect to experimental therapeutic cancer research, there are several methodologies that could be employed for the identification of cytotoxic activities, among which colourimetric absorbance assay studies are cheap, simple and convenient strategies, when compared 
with other radioactive and non-radioactive approaches. [3(4,5-dimethylthiazol-2-yl)-5-(3-carboxymethoxyphenyl)-2(4-sulfophenyl)-2H-tetrazolium] (MTS) assay is one of the commonly employed methods, based on the one-step direct addition of the testing agents [both MTS and phenazine methosulfate (PMS)] to the cultured cells for colour development. This MTS assay has been employed in the identification of several putative antitumor agents, such as, for instance, novel cantharidin analogues exhibiting anticancer activity under in vitro conditions (2-5).

Both organic and organometallic compounds have been extensively explored for the possible cytotoxic activity towards cancer cells $(6,7)$. In addition, inorganic ions, especially transition metal series with variable oxidation number, have been demonstrated to retain cytotoxic potential on cancer cells (8). Of these, iron ions show two common oxidation states that include the ferrous ions [iron $(+2)]$ and ferric ions [iron (+3)] respectively; both of which were demonstrated to be cytotoxic in vitro in the form of iron (II) sulphate and iron (III) chloride (9). Itoh et al showed that $100 \mu \mathrm{M}$ iron ions were sufficient to induce cell death, after $3 \mathrm{~h}$ of treatment, in murine hepatoma cells, but not in normal murine hepatocytes. Here we have performed experiments to evaluate the possible antiproliferative activity of iron (II) sulphate on human cancer cell lines, employing three different biochemical methods, combining these with cellular morphology assays. Different results were observed using the employed different approaches.

\section{Materials and methods}

Cell lines and cell culture conditions. The human chronic myelogenous leukaemia cell line K562 (CCL-243) and the human breast carcinoma T47D (HTB-133) cell line were obtained from American Type of Culture Collection. Cells were maintained in minimum essential medium (Sigma Chemicals) supplemented with $10 \%$ heat inactivated fetal bovine serum (Invitrogen), together with antibiotics including penicillin and streptomycin. Cells were allowed to grow in a humidified cell culture incubator in 5\% carbon dioxide. Iron (II) sulphate stock solution was prepared freshly each time at a concentration of $1 \mathrm{M}$ by dissolving in sterile distilled water and at least a 1000 -fold dilution was used in the cell culture experiment.

MTS assay. Changes in the cellular viability of iron (II) sulphate-treated cells were monitored using the MTS activity assay as reported previously (2-5). Briefly, both K562 CML cells and T47D breast carcinoma cells at a concentration of $1 \times 10^{5}$ per ml were seeded at day 0 . After $24 \mathrm{~h}$, medium without phenol (as phenol red would interfere with development of the brownish colour from the reduced MTS) was changed and freshly prepared iron (II) sulphate was added at different concentrations. After 48 h of incubation, MTS (Promega)/ PMS (Sigma) as electron coupling agent mixed solution was added directly into the wells and further incubated for brown colour development. Afterwards, optical absorbance was determined at $490 \mathrm{~nm}$ using a microplate reader according to the user manual.

3-(4,5-dimethylthiazol-2-yl)-2,5-diphenyltetrazolium bromide (MTT) assay. Briefly, MTT assay was performed similarly to
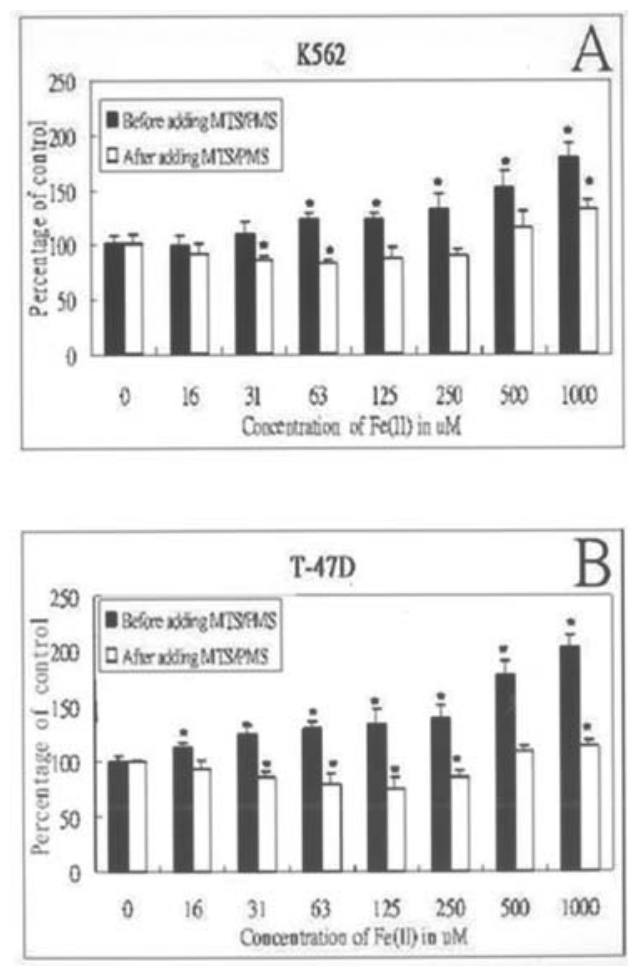

Figure 1. Influence of 48-h treatment with iron (II) sulphate on the growth of (A) K562 CML cells and, (B) T-47D breast carcinoma cells, measured before and after MTS/PMS addition. Each experiment was performed in triplicate and three independent experiments were performed, obtaining similar results. Results represent one among three independent experiments. Each reading was subtracted by the mean of blanks without cell and iron (II) sulphate but with culture medium and MTS/PMS ( ${ }^{*} \mathrm{p}<0.05$ when compared with untreated control).

the MTS assay described before. The major difference was that after incubating MTT $(5 \mathrm{mg} / \mathrm{ml}$ dissolved in phosphate buffered saline, both from Sigma) with T47D cancer cells, cell culture medium was removed from the wells and dimethyl sulfoxide (Sigma) was added instead of a direct reading of the culture plate after adding the MTS/PMS, as mentioned previously. Finally, absorbance was determined at $595 \mathrm{~nm}$ using a microplate reader.

Adenosine triphosphate (ATP) determination. Briefly, the methodology for determination of ATP content as a measurement of cellular growth was the same as described elsewhere (10). Here we used an ATP quantitation kit purchased from Promega. The resulting ATP content was determined by chemiluminescence detection using a microplate reader.

Morphological monitoring of iron (II) sulphate-treated T47D cells. Any morphological changes associated with iron (II) sulphate treatment of T47D breast cancer cells were recorded under an inverted microscope at the scheduled time points after fixing the cells with ethanol and staining with methylene blue.

Statistical analysis. Student's t-test was used in data analysis, and the results were considered as statistically significant when $\mathrm{p}$ values were $<0.05$ when treated samples were compared with untreated controls. 

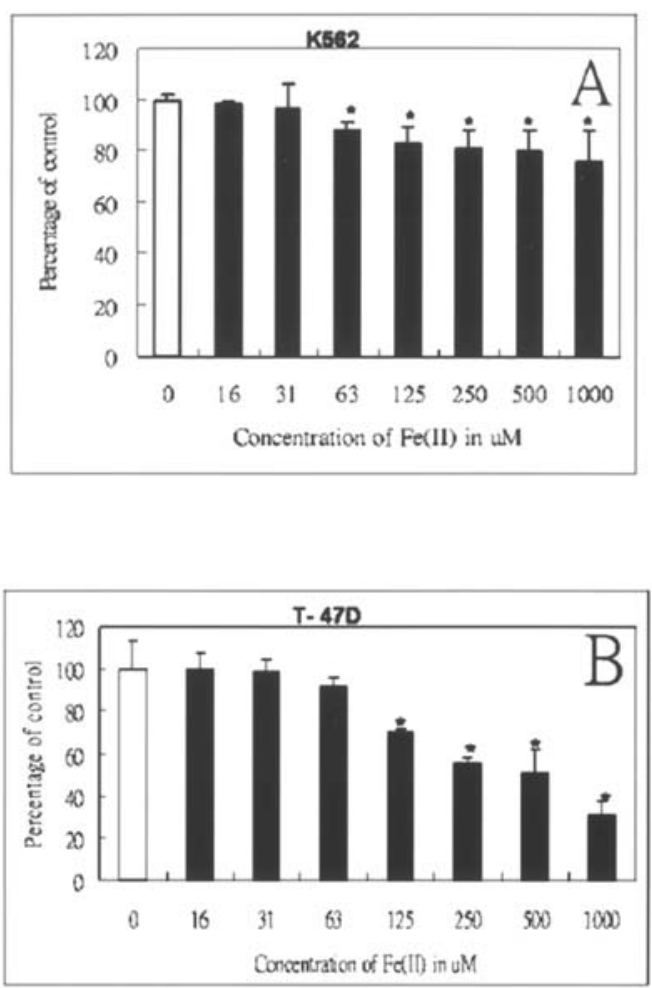

Figure 2. (A) Influence of 48-h treatment with iron (II) sulphate on the growth of K562 cells, measured by ATP content. Each reading was subtracted by the mean of blanks without cell and iron (II) sulphate but with culture medium and reaction buffer for ATP detection. (B) Influence of 48-h treatment with iron (II) sulphate on the growth of T-47D cells, measured by MTT assay. Each reading was subtracted by the mean of blanks without cell and iron (II) sulphate but with dimethyl sulfoxide only at $595 \mathrm{~nm}$. For both $\mathrm{A}$ and $\mathrm{B}$, each experiment was performed in triplicate and three independent experiments were performed, obtaining similar results. Results represent one among three independent experiments $\left({ }^{*} \mathrm{p}<0.05\right.$ when compared with untreated control).

\section{Results}

Iron (II) sulphate showed an inhibitory and then a proliferative effect on both K562 and T47D cancer cells after MTS assay. We have determined that Fe (II) sulphate appears to exhibit both growth inhibitory as well as growth stimulatory effects on the two human cancer cell lines, K562 and T47D, when the test is based on the MTS assay and 48-h incubations are performed at concentrations ranging from 16-1000 $\mu \mathrm{M}$. The results obtained are shown in Fig. 1A and B. We found that iron (II) sulphate exhibits an inhibitory effect when used at 16to $125-\mu \mathrm{M}$ concentrations. On the contrary, iron (II) sulphate appeared to be growth stimulatory when its concentration was further increased to $1000 \mu \mathrm{M}$, and the MTS assays were performed. In addition, we also determined the optical absorbance at $490 \mathrm{~nm}$ after iron (II) sulphate incubation for $48 \mathrm{~h}$ but before MTS/PMS addition; interestingly, a dosedependent increment was also observed as shown in Fig. 1A and $\mathrm{B}$, suggesting that the cell treatment might strongly interfere with optical absorbance at $490 \mathrm{~nm}$.

Iron (II) sulphate showed a consistent growth inhibitory effect on K562 CML cells and T47D breast cancer cells when examined by ATP content assay and MTT assay respectively.

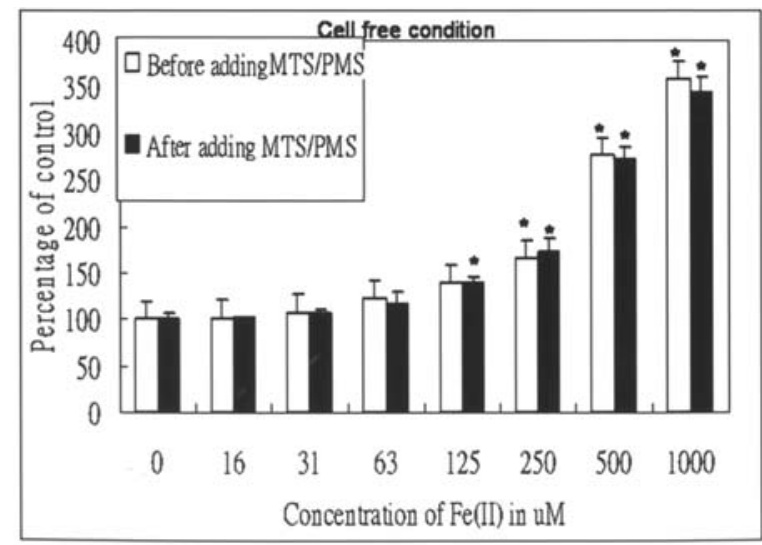

Figure 3. Influence of iron (II) sulphate on the changes of optical absorbance at $490 \mathrm{~nm}$. Influence of 48-h treatment with iron (II) sulphate on the growth of K562 cells, measured by ATP content. Each experiment was performed in triplicate and three independent experiments were performed, obtaining similar results. Results are shown as one of the three independent experiments. The mean optical absorbance at $490 \mathrm{~nm}$ for the iron (II) sulphate free samples was considered as $100 \%$ control ( ${ }^{*}$ p value $<0.05$ when compared with untreated control).

We repeated the iron (II) sulphate incubation experiment on K562 CML cells and monitored the effects by using the ATP content assay. The results obtained are shown in Fig. 2 A. Surprisingly, but in agreement with the MTS assay performed on cells treated with 16 - to $125-\mu \mathrm{M}$ concentrations, a dosedependent growth inhibitory effect with increasing iron (II) sulphate concentration was observed.

In order to confirm these data, we further repeated the iron (II) sulphate experiment on T47D breast cancer cells and performed an MTT assay. Results are shown in Fig. 2B, demonstrating an even stronger dose-dependent anti-proliferative effect with increasing iron (II) sulphate concentration.

A consistent increment of absorbance at $490 \mathrm{~nm}$ was observed with increased iron (II) sulphate concentration either in the absence or presence of MTS/PMS reagents even in a cell-free environment. Since the different assay methods employed showed controversial results, we determined whether the growth-stimulatory effect of iron (II) sulphate, when used at a high concentration and assayed by MST/PMS test, was actually a false positive phenomenon. To this aim, we repeated the experiment again to see if there was any influence of iron (II) sulphate in a cell-free environment, in the presence or absence of MTS/PMS. Fig. 3 shows that there was a consistent increment of the absorbance at $490 \mathrm{~nm}$ observed with increased iron (II) sulphate concentration, either in the presence or absence of MTS/PMS reagents, even in a cell-free environment.

Iron (II) sulphate was actually cytotoxic when administered to T47D breast cancer cells at a $1000-\mu \mathrm{M}$ concentration. The morphological changes of T47D breast cancer cells treated with $1000-\mu \mathrm{M}$ iron (II) sulphate for $48 \mathrm{~h}$ were investigated under an inverted microscope after fixing the cells with ethanol and staining with methylene blue. Fig. 4 clearly shows that iron (II) sulphate is cytotoxic towards T47D cancer cells at a high concentration $(1000 \mu \mathrm{M})$ as loss of adherent property, cell rounding and shrinkage were observed. 

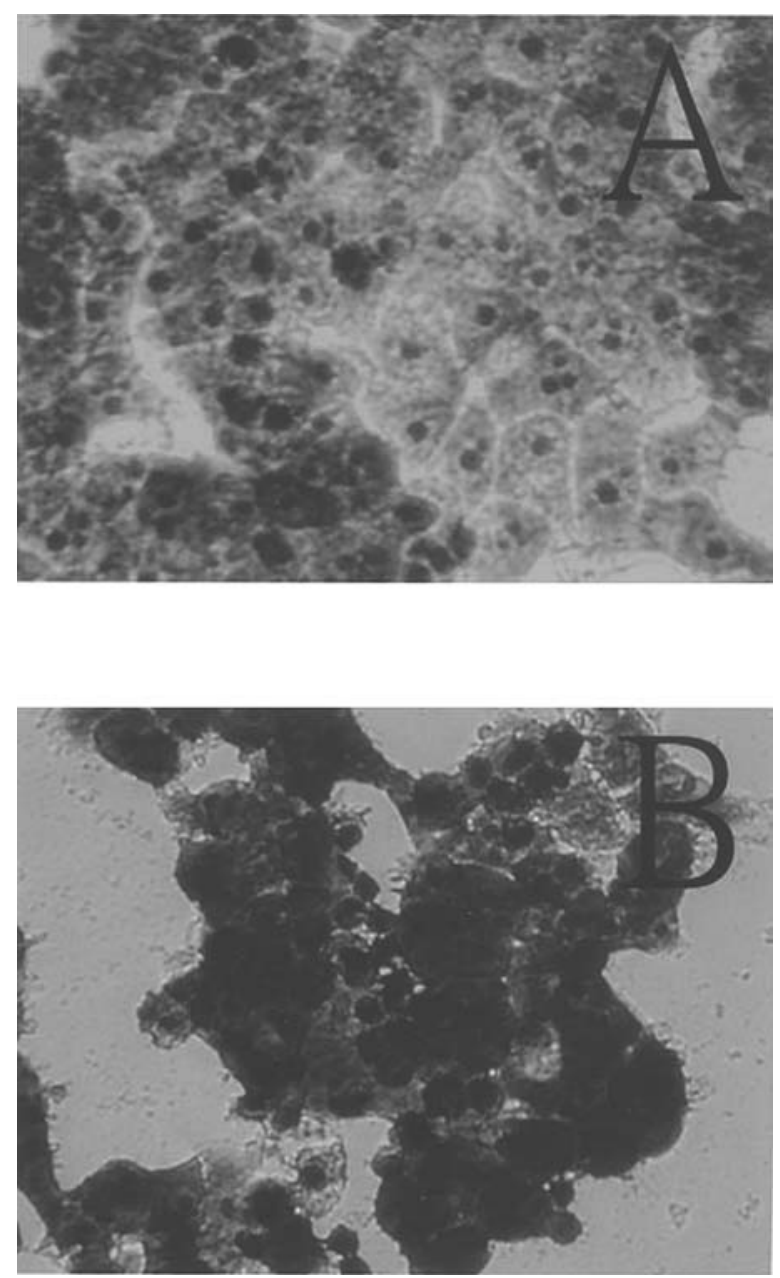

Figure 4. Morphological investigation of T-47D breast cancer cells after $48 \mathrm{~h}$ of culture in the absence (A) and in the presence (B) of $1000-\mu \mathrm{M}$ iron (II) sulphate.

\section{Discussion}

It is well known that many transitional metal ions are cytotoxic towards human cancer cells. Examples include vanadium, chromium and iron. When concerning vanadium, Lampronti et al discovered that vanadium (V) and (IV) complexes including $\mathrm{NaVO}_{3}, \mathrm{~V}_{2} \mathrm{O}_{5}, \mathrm{Na}_{6} \mathrm{~V}_{10} \mathrm{O}_{28}, \mathrm{Na}\left[\mathrm{VO}_{2}\right.$ (Hhida) $4 \mathrm{H}_{2} \mathrm{O}$, $\mathrm{VOSO}_{4} \cdot 4 \mathrm{H}_{2} \mathrm{O}, \mathrm{VO}(\mathrm{acac})_{2}, \mathrm{VO}\left(\mathrm{acac}-\mathrm{NMc}_{2}\right)_{2}, \mathrm{VO}\left(\mathrm{OH}_{2}\right)$ (Hhida). $\mathrm{CH}_{3} \mathrm{OH}$ and $\mathrm{V}_{2} \mathrm{O}_{2}(\mu . \mathrm{O})(\text { Hhida })_{2} \cdot \mathrm{H}_{2} \mathrm{O}$ were all antiproliferative towards $\mathrm{K} 562 \mathrm{CML}$ cells, when administered for $96 \mathrm{~h}$ at concentrations ranging from 15-1500 $\mu \mathrm{M}$. Inhibition of K562 cell growth was not associated with erythroid differentiation, but with activation of apoptosis (8). In considering chromium, it was found that chromium (VI) could readily induce oxidative stress, lipid oxidation, nucleic damage and programmed cell death in both in vitro and in vivo models including K562 CML and murine macrophage A774A.1 cells as well as an experimental mouse model system (11). In these experiments concentrations ranging from $0.6 \mu \mathrm{M}$ and $25 \mu \mathrm{M}$ where used in 48-h treatments. Even chromium (VI) was shown to have stronger toxic effect when compared with chromium (III), perhaps the safety of chromium (III) was mainly due to the nature of its corresponding ligands. Iron
(II) is also reported to have cytotoxic activity on murine hepatoma but not normal murine hepatocytes. The mitochondrial DNA was markedly damaged after incubating with iron (II) at a concentration of $100 \mu \mathrm{M}$ for $3 \mathrm{~h}$. It was believed that the major mechanism was the lower level of superoxidase dismutase in rat hepatoma cells when compared with nontransformed cells. Therefore it is believed that iron (II) at a high concentration is cytotoxic towards cancer cells (9).

Here we have performed experiments to evaluate whether iron (II) sulphate is a true anti-proliferative and cytotoxic agent on human cancer cell lines, after comparison of three different biochemic methods as well as cellular morphology determinations. We used as experimental models two human cancer cell lines, the CML K562 and the breast carcinoma T47D cell lines.

We first analysed the possible growth inhibitory effects of iron (II) sulphate on these two cancer cell lines by the MTS assay. We found that iron (II) sulphate exhibits an inhibitory effect when used at concentrations ranging between 16 and $125 \mu \mathrm{M}$; on the contrary, iron (II) sulphate became surprisingly growth stimulatory when its concentration was further increased to $1000 \mu \mathrm{M}$. In addition, a dose-dependent increase in absorbance was observed when we repeated the experiments at the same wavelength without the addition of MTS/PMS. This suggested that the increase in absorbance in the presence of high concentrations $(1000 \mu \mathrm{M})$ of iron (II) sulphate might not be due to the redox reaction between the cellular dehydrogenase and MTS/PMS. When we further repeated the assay using adenosine triphosphate content detection and MTT assay, iron (II) sulphate showed a consistent dose-dependent growth inhibitory effect on both K562 and T47D cells. Therefore, the conclusion that iron (II) sulphate is growth stimulatory on both cancer cell lines at higher concentrations is unlikely. When we performed morphological investigation after methylene blue staining, there was a clear demonstration that iron (II) sulphate at a concentration of $1000 \mu \mathrm{M}$ is cytotoxic to T47D cells. In the case of K562 CML cells, we also observed a certain degree of cell shrinkage but it was not as evident as in T47D (data not shown). Finally, a consistent increment of the absorbance at $490 \mathrm{~nm}$ was observed with increased iron (II) sulphate concentration in a cell-free environment, either in the presence or in the absence of MTS/PMS. Thus we believe that iron (II) sulphate is actually growth inhibitory and cytotoxic at high concentrations towards both K562 and T47D cancer cell lines. The paradoxical growth stimulating activity found only using the MTS assay was solely due to the oxidation of initial pale green iron (II) to brownish iron (III) at the highest concentrations used during incubation in the aqueous condition; accordingly with our interpretation, the change in colour of the iron (III) ion form during the continuous oxidation of iron (II) was responsible for the masking of the decrease in brown colour originally contributed by the low-level cellular metabolic activity. Such misinterpretation could easily be avoided if the original culture medium is removed before the addition of MTS/PMS. Therefore, it is suggested that special precautions should be taken when studying growth stimulatory or growth inhibitory assays using MTS/PMS if medium is not removed before the addition of the MTS/PMS reagents.

It is widely accepted that there is an urgent need for novel therapeutic agents for cancer treatment and in vitro screening 
is the first step to select potential candidates for further investigations. However, in vitro drug screening may not be as simple as expected as mentioned by Etique et al when ethanol was used as a solvent for experimental therapeutic research when MCF-7 breast cancer cells were employed as the experimental tool (12). In conclusion, basic fundamental chemistry knowledge is an essential and necessary criterion for those who are working in the field of drug discovery and development.

\section{Acknowledgements}

We acknowledge the support by the Area of Strategic Development (ASD) programme of the Hong Kong Polytechnic University (H.K.P.U.) (work programme A012). This study was supported by the Areas of Excellence Scheme established under the University Grants Committee of the Hong Kong Special Administrative Region, P.R. China (Project No. AoEP-10/01) and a Niche Area Grant offered by the H.K.P.U. to Dr F. Cheung (grant number BB8R; HK\$ 100,000). Drs F.Y. Lau and C.H. Chui are the honourary tutors kindly offered by Professors J.J.Y. Sung and G.Y.M. Cheng from Department of Medicine and Therapeutics, Prince of Wales Hospital, The Chinese University of Hong Kong. Dr S.H.L. Kok is supported by the post of 'Research Fellow' kindly offered by Professor A.S.C. Chan. Dr C.H. Chui is supported by the funding from the 'Lo Ka Chung Centre for Natural Anti-Cancer Drugs Development' that was kindly donated by Mr. Lo Ka Chung. Dr C.H. Chui would like to thank Professor R. Gambari for his constructive criticisms and suggestions. Lastly, Dr S.H.L. Kok would like to thank Professor A.S.C. Chan and Dr J.C.O. Tang as well as all the staff and students in his laboratory for their support and encouragement for the past 8 years.

\section{References}

1. Mayr LM and Boettcher A: Miniaturisation of assay development and screening. Drug Discov World 7: 17-27, 2006

2. Kok SHL, Chui CH, Lam WS, Chen J, Lau FY, Cheng GYM, Wong RSM, Lai PBS, Leung TWT, Tang JCO and Chan ASC: Apoptotic activity of a novel synthetic cantharidin analogue on hepatoma cell lines. Int J Mol Med 17: 945-949, 2006.

3. Kok SHL, Chui CH, Lam WS, Chen J, Lau FY, Wong RSM, Cheng GYM, Tang WK, Cheng CH, Tang JCO and Chan ASC: Mechanistic insight into a novel synthetic cantharidin analogue in a leukaemia model. Int J Mol Med 18: 375-380, 2006.

4. Kok SHL, Chui CH, Lam WS, Chen J, Lau FY, Wong RSM, Cheng GYM, Tang WK, Teo ITN, Cheung F, Cheng CH, Chan ASC and Tang JCO: Apoptogenic activity of a synthetic cantharimide in leukaemia: Implication on its structural activity relationship. Int J Mol Med 18: 1217-1221, 2006.

5. Kok SHL, Chui CH, Lam WS, Chen J, Lau FY, Wong RSM, Cheng GYM, Lai PBS, Leung TWT, Tang JCO and Chan ASC: Synthesis and structure evaluation of a novel cantharimide and its cytotoxicity on hepatoma cell line. Bioorg Med Chem Lett 17: 1155-1159, 2007.

6. Kok SHL, Gambari R, Chui CH, Lam WS, Chen J, Lau FY, Wong RSM, Cheng GYM, Lai PBS, Leung TWT, Chan ASC and Tang JCO: A mini-review for the in vitro anti-cancer property of five novel synthetic cantharidin analogues. Minerva Biotec 18: 153-157, 2006.

7. Messere A, Fabbri F, Borgatti M, Gambari R, Blasio BD, Pedone $\mathrm{C}$ and Romanelli A: Antiproliferative activity of Pt(II) and (Pd)II phosphine complexes with thymine and thymidine. J Inorg Biochem 101: 254-260, 2007.

8. Lampronti I, Bianchi N, Borgatti M, Fabbri E, Vizziello L, Khan MT, Ather A, Brezena D, Tahir MM and Gambari R: Effects of vanadium complexes on cell growth of human leukemia cells and protein-DNA interactions. Oncol Rep 14: 9-15, 2005.

9. Itoh H, Shioda T, Matsura T, Koyama SY, Nakanishi T, Kajiyama $\mathrm{G}$ and Kawasaki T: Iron ion induces mitochondrial DNA damage in HTC rat hepatoma cell culture - Role of antioxidants in mitochondrial DNA protection from oxidative stresses. Arch Biochem Biophys 313: 120-125, 1994.

10. Kok SHL, Chui CH, Lam WS, Chen J, Tang JCO, Lau FY, Cheng GYM, Wong RSM and Chan ASC: Induction of apoptosis on carcinoma cells by two synthetic cantharidin analogues. Int $\mathbf{J}$ Mol Med 17: 151-157, 2006.

11. Bagchi D, Stohs SJ, Downs BW, Bagchi M and Preuss HG: Cytotoxicity and oxidative mechanisms of different forms of chromium. Toxicology 180: 5-22, 2002.

12. Etique N, Chardard D, Chesnel A, Merlin JL, Flament S and Grillier-Vuissoz I: Ethanol stimulates proliferation, ERalpha and aromatase expression in MCF-7 human breast cancer cells. Int J Mol Med 13: 149-155, 2004. 